\title{
Rate of Nitration of Benzene with Mixed Acid
}

\author{
R. D. BIGGS and R. R. WHITE
}

University of Michigan, Ann Arbor, Michigan

The rates of nitration of benzene by nitric acid in mixed acid to produce mononitrobenzene have been measured in well-emulsified reaction mixtures in the temperature range from $34^{\circ}$ to $54^{\circ} \mathrm{C}$. The acid-phase compositions ranged from 1.6 mole $\%$ nitric acid and 27 mole \% sulfuric acid to 35 mole \% nitric acid and zero \% sulfuric acid; the organicphase composition ranged from 4 to 95 mole $\%$ benzene, and the relative extent of the acid and organic phases was varied from 25 to 80 volume \% acid phase.

The reaction rate based on the total volume of the reacting mixture is shown to be a function of the phase compositions, temperature, and volume \% of acid phase.

The nitration of aromatic hydrocarbons has been the subject of numerous investigations since nitrobenzene was first synthesized in 1834 from benzene and fuming nitric acid (13). However, only a few recent investigators have determined data which are useful for engineering applications $(3,4,8,9,11,12,14)$. The uncertainties inherent in the reaction-rate data available at the present time led to this investigation of the variables affecting the rate of nitration of benzene in a continuous, stirred-pot reaction system.

\section{EXPERIMENTAL EQUIPMENT}

A continuous-flow, stirred-pot reaction system was used to study the variables affecting the rate of nitration of benzene with mixed acid. This type of apparatus possesses certain advantages that a batch reaction system does not have: that is, (1) large samples can be collected without affecting the reacting system, and (2) neither the reaction rate nor the reactor compositions change with time when steady state conditions are achieved, the reaction rate being calculated from the steady state material balance. Figure 1 is a flow diagram of the apparatus.

As shown in Figure 1, mixed acid and benzene flowed by gravity from the constant-head reservoirs (12), $A$ and $B$, through the flow meters $C$ and $D$ and dripped into the feed lines $E$ and $F$. The Pyrex-glass capillary-tube flow meters $C$ and $D$ were used to indicate instantaneous flow rates of the benzene and acid feeds for control purposes. The feeds drained through the feed lines into the reactor $G$. The stirrer,

R. D. Biggs is with Ethyl Corporation, Baton Rouge, Louisiana

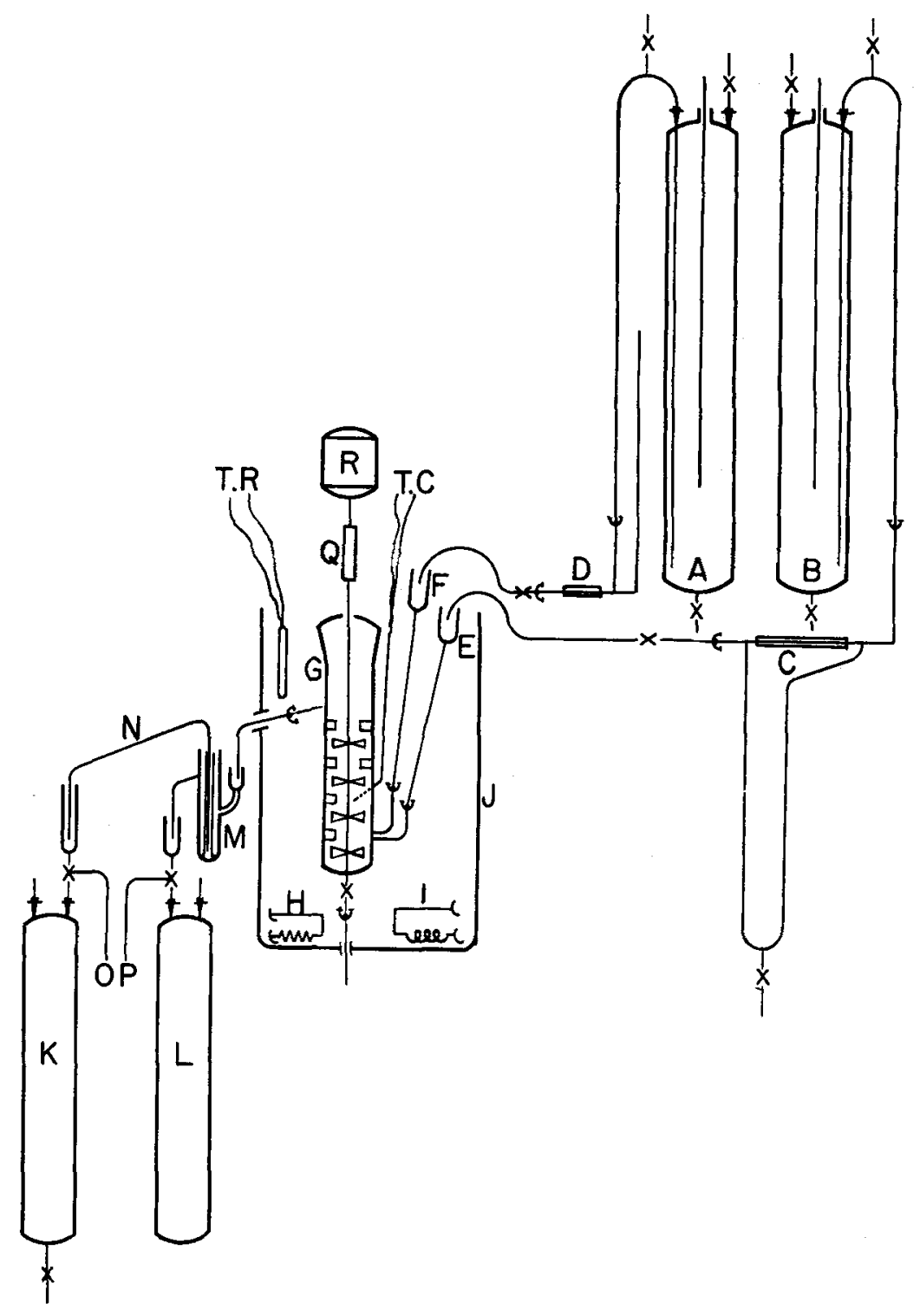

Fig. 1. Schematic diagram of equipment. 
driven by a variable-speed motor $R$ through a sleeve bearing $Q$, kept the acid and organic phases in the reactor well emulsified. The product from the reactor overflowed into the separator $M$, where the two phases separated completely. The lighter organic phase rose to the top in the separator and overflowed into the organic-product receiver $L$. The heavier acid phase settled to the bottom in the separator, where it was drained by the syphon tube $N$ into the acid-product reservoir $K$.

A large glass battery jar $J$ contained water which was maintained at a constant tem-
With the exception of the stirrer assembly in the reactor all the apparatus was constructed of glass. Flow rates were determined by measuring the difference in volume over a measured period of time in each of the calibrated reservoirs. Samples of the feed streams were taken from the drain in the bottom of each feed reservoir. Product samples were taken through the side tubes $O$ and $P$.

A detailed drawing of the reactor is shown in Figure 2. The reactor was constructed of Pyrex glass and was designed to maintain a uniform emulsion of the acid and organic cal Company were mixed with distilled water to obtain the desired feed-acid concentration. The nitric acid was water white and contained no lower oxides of nitrogen detectable by chemical analysis.

Reagent-grade benzene manufactured by the General Chemical Division of Allied Chemical and Dye Corporation was used in all the experiments. The minimum freezing point reported by the manufacturer corresponds to a minimum purity of 99.53 mole $\%$.

Table 1. Operating Conditions

\begin{tabular}{|c|c|c|c|c|c|c|c|c|}
\hline \multirow[b]{2}{*}{ Run } & \multirow{2}{*}{$\begin{array}{c}\text { Reaction } \\
\text { liquid } \\
\text { volume, } \\
\text { Mi }\end{array}$} & \multicolumn{2}{|c|}{$\begin{array}{l}\text { Acid phase in } \\
\text { reaction mixture }\end{array}$} & \multirow{2}{*}{$\begin{array}{l}\text { Acid } \\
\text { feed } \\
\text { rate, } \\
\text { g. } / \mathrm{hr} \text {. }\end{array}$} & \multirow{2}{*}{$\begin{array}{l}\text { Benzene } \\
\text { feed } \\
\text { rate, } \\
\text { g. } / \text { hr. }\end{array}$} & \multirow{2}{*}{$\begin{array}{l}\text { Acid } \\
\text { product } \\
\text { rate, } \\
\text { g. } / \mathrm{hr} \text {. }\end{array}$} & \multirow{2}{*}{$\begin{array}{c}\text { Organic } \\
\text { product } \\
\text { rate, } \\
\text { g. } / \mathbf{h r} .\end{array}$} & \multirow{2}{*}{$\begin{array}{c}\text { Reaction rate, } \\
\text { g.-mole/ } \\
\text { (hr.)(liter) } \\
\text { total vol. }\end{array}$} \\
\hline & & $\begin{array}{l}\text { Reactor } \\
\text { vol., } \%\end{array}$ & $\begin{array}{l}\text { Overflow } \\
\text { vol., } \%\end{array}$ & & & & & \\
\hline $1^{*}$ & 416 & 59.2 & 58.5 & 690 & 228 & 625 & 301 & 3.17 \\
\hline $2^{*}$ & 396 & 73.5 & 58.2 & 674 & 211 & 594 & 291 & 3.54 \\
\hline $3^{*}$ & 403 & 58.8 & 58.4 & 585 & 191 & 521 & 252 & 2.98 \\
\hline $4^{*}$ & 413 & 56.4 & 58.0 & 642 & 205 & 564 & 277 & 3.08 \\
\hline $5^{*}$ & 402 & 56.1 & 58.8 & 660 & 212 & 600 & 285 & 3.22 \\
\hline $6^{*}$ & 404 & 57.4 & 57.5 & 1,103 & 431 & 1,067 & 462 & 1.398 \\
\hline $7^{*}$ & 405 & 59.5 & 60.8 & 686 & 234 & 667 & 255 & 0.957 \\
\hline $8^{*}$ & 341 & 30.2 & 33.5 & 294 & 288 & 270 & 314 & 1.323 \\
\hline $9^{*}$ & 350 & 40.0 & 41.8 & 401 & 266 & 358 & 302 & 1.780 \\
\hline 10 & 355 & 51.2 & 53.5 & 596 & 219 & 516 & 306 & 4.57 \\
\hline 11 & 345 & 52.7 & 54.0 & 609 & 214 & 519 & 303 & 4.86 \\
\hline 12 & 350 & 62.0 & 63.4 & 729 & 239 & 714 & 249 & 0.249 \\
\hline 13 & 324 & 55.6 & 54.8 & 487 & 217 & 457 & 254 & 1.175 \\
\hline 14 & 323 & 50.8 & 53.5 & 416 & 215 & 404 & 233 & 0.503 \\
\hline 15 & 322 & 58.7 & 58.0 & 570 & 253 & 562 & 265 & 0.265 \\
\hline 16 & 327 & 60.4 & 60.4 & 772 & 284 & 753 & 304 & 0.575 \\
\hline 17 & 344 & 58.2 & 62.2 & 534 & 194 & 527 & 211 & 0.771 \\
\hline 18 & 328 & 42.6 & 46.8 & 443 & 274 & 433 & 289 & 0.666 \\
\hline 19 & 336 & 78.6 & 78.2 & 940 & 139 & 927 & 164 & 1.155 \\
\hline 20 & 324 & 35.5 & 42.1 & 302 & 229 & 299 & 240 & 0.468 \\
\hline 21 & 323 & 25.0 & 31.7 & 302 & 345 & 287 & 357 & 0.470 \\
\hline 22 & 337 & 60.8 & 63.5 & 567 & 175 & 549 & 198 & 1.138 \\
\hline 23 & 328 & 65.5 & 64.4 & 575 & 184 & 551 & 213 & 0.995 \\
\hline 24 & 318 & 64.4 & 63.4 & 429 & 159 & 426 & 162 & 0.0648 \\
\hline 25 & 332 & 55.4 & 56.8 & 561 & 198 & 503 & 254 & 2.91 \\
\hline 26 & 324 & 61.1 & 60.4 & 544 & 194 & 534 & 206 & 0.424 \\
\hline 27 & 312 & 64.1 & 61.8 & 506 & 185 & 494 & 194 & 0.289 \\
\hline 28 & 334 & 54.5 & 54.5 & 548 & 197 & 473 & 274 & 4.57 \\
\hline 29 & 326 & 52.8 & 53.0 & 501 & 203 & 434 & 264 & 2.73 \\
\hline 30 & 341 & 70.7 & 72.3 & 741 & 118 & 670 & 192 & 3.70 \\
\hline 31 & 306 & 26.1 & 28.1 & 324 & 405 & 294 & 443 & 2.09 \\
\hline 32 & 312 & 32.7 & 36.8 & 421 & 319 & 376 & 368 & 2.69 \\
\hline 33 & 339 & 77.6 & 80.2 & 806 & 80.6 & 747 & 142 & 2.86 \\
\hline 34 & 299 & 29.8 & 35.5 & 481 & 412 & 431 & 472 & 3.33 \\
\hline
\end{tabular}

*Runs 1 to 9 were made with old reactor.

perature by circulating cooling water through the coils $I$ and by intermittently heating with an electric heater $H$. The reactor temperature was measured by a copper-constantan thermocouple $T C$ which extended into the center of the reactor. phases and a high degree of circulation of the contents.

\section{MATERIALS}

Reagent-grade sulfuric and nitric acids manufactured by the J. T. Baker Chemi-

\section{EXPERIMENTAL PROCEDURE}

In each experimental run the reaction system was brought to steady state conditions by operation for about $21 / 2 \mathrm{hr}$. at a constant reactor temperature and with constant feed rates. The approach of the or- 
ganic-product density to a constant value within $\pm 0.1 \%$ served as an indication of the approach to steady state conditions.

After steady state conditions had been achieved, samples of the acid- and organicproduct streams were collected for density measurement and analysis. After the samples were collected, the total volume of the emulsion and the volume of the acid phase in the reactor were measured.
Standard methods for analysis were used for the total acid, nitrous acid (15), and nitric acid (10). The sulfuric acid was calculated as the difference between the percentage of total acid and the percentage of nitric acid. The organic-product sample was washed with water, and the benzene was determined by density measurement after the sample was dried with calcium chloride. A qualitative test was made for dinitro- varied from 1,290 to $2,810 \mathrm{rev} . / \mathrm{min}$., the volume percentage of acid phase from 25 to 79 , and the temperature from $34.6^{\circ}$ to $53.7^{\circ} \mathrm{C}$. The acid-phase composition was varied from 35 mole \% nitric acid and $0 \%$ sulfuric acid to 1.6 mole $\%$ nitric acid and 27 mole $\%$ sulfuric acid. The organicphase composition ranged from-4, to 97 mole \% benzene.

Table 2. Analytical Data

Acid feed composition, Acid-product composition, mole $\%$

$\begin{array}{cr}\mathrm{HNO}_{3} & \mathrm{H}_{2} \mathrm{SO} \\ 15.4 & 23.8 \\ 15.0 & \mathbf{2 4 . 5} \\ 15.1 & 24.6 \\ 15.0 & 24.7 \\ 15.0 & 24.7 \\ 5.72 & 24.9 \\ 5.69 & 24.7 \\ 12.1 & \mathbf{2 4 . 8} \\ 12.1 & 24.8 \\ 18.0 & 27.1\end{array}$

18.0

15.5

35.7

38.9

36.2

23.3

9. 70

9.64

9. 39

9.73

9.41

9. 73

39.0

29.7

13.9

\section{9}

24.3

15.6

19.6

15.3

\section{4}

15.4

14.2

16.1

mole \%

$\begin{array}{cc}\mathrm{HNO}_{3} & \mathrm{H}_{2} \mathrm{SO}_{4} \\ 5.36 & 24.1 \\ 4.70 & 24.9 \\ 4.64 & 24.9 \\ 4.59 & 24.9 \\ 4.64 & 25.1 \\ 3.32 & 24.9 \\ 3.08 & 24.6 \\ 4.11 & 25.0 \\ 4.17 & 25.0 \\ 3.06 & 27.2 \\ & \end{array}$

27.1

14.0

4.29

0

10.1

21.0

21.0

20.7

20.9

21.2

21.2

0

23.1

15.0

6. 69

26.3

17.6

24.8

24.8

24.8

24.5

24.8

$\begin{array}{rr}3.15 & 27.2 \\ 14.7 & 14.0 \\ 30.5 & 4.1 \\ \mathbf{3 5 . 1} & 0 \\ 34.3 & 0\end{array}$

27.2

14.0

4.19

0 Organic-product composition,
mole $\%$

$\begin{array}{ccc}21.4 & 10.1 & 4.70 \\ 7.07 & 21.1 & 3.18 \\ 7.19 & 21.1 & 2.45 \\ 7.38 & 20.9 & 5.78 \\ 7.06 & 20.7 & 2.14 \\ & & \\ 7.00 & 22.2 & 1.96 \\ 6.11 & 21.0 & 4.06 \\ 34.2 & 0 & 9.08 \\ 29.1 & 0 & 1.88 \\ 4.96 & 23.4 & 7.06 \\ & & \\ 12.4 & 15.1 & 3.42 \\ 23.6 & 6.49 & 3.52 \\ 1.59 & 26.6 & 4.14 \\ 9.83 & 18.1 & 11.0 \\ 5.84 & 25.0 & 15.9 \\ & & \\ 4.38 & 25.6 & 2.90 \\ 4.66 & 25.2 & 4.14 \\ 7.43 & 24.2 & 21.3 \\ 5.03 & 25.2 & 4.36\end{array}$

$\mathrm{HNO}_{3}$
9.21
8.91
8.69
8.22
8.31
1.76
1.85
2.52
3.45
7.37

7.80
2.92
10.2
5.79
3.79

4.70
3.18
2.45
5.78
2.14

1.96
4.06
9.08
1.88
7.06

3.42
3.52
4.14
11.0
15.9

2.90
4.14
21.3
4.36

$\mathrm{H}_{2} \mathrm{SO}_{4} *$

$\mathrm{C}_{6} \mathrm{H}_{6}$

$\mathrm{C}_{6} \mathrm{H}_{5} \mathrm{NO}_{2}$

Benzene, mole $\%$ acid-free basis

trace

0.005

48.8

42.0

53.8

trace 44.8

$0.052 \quad 47.1$

473

46.5

44.6

$0.062 \quad 47.6$

44.0

48.1

49. 1

51.4

52.0

0.033

88.2

$0.028 \quad 85.5$

$0.054 \quad 85.4$

9. 99

12.6

12.0

78.8

0.068

0.029

39.0

17.7

53.6

89.8

87.2

87.7

81.7

41.7

$0.010 \quad 35$

$0.019 \quad 94.2$

$0.027 \quad 77.5$

0

0

88.7

56.4

2.87

38.8

12.3

5.56

97.0

86.3

94.2

$2.53 \quad 97.4$

0.026

0.034

0.008

0.033

0.022

90.4

4.92

94.8

86.4

91.5

73.8

92.8

10.4

6.06

20.4

89.3

5.06

78.4

94.8

$\begin{array}{ll}\text { trace } & 94.7 \\ \text { trace } & 79.5 \\ 0 & 78.3 \\ 0 & 97.1 \\ \text { trace } & 57.5\end{array}$

3.38

96.4

16.4

12.6

82.9

1.00

86.2

35.4

99.0
61.9

0.052

91.2

trace

trace

trace

0.086

92.9

38.3

58.7

13.4

\subsection{3}

94.5

3.56

96.2

57.6

30.3

70.7

65.9

15.9

$0.012 \quad 85.1$

trace $\quad 76.3$

0.016

trace

12.0

74.4

18.1
87. 6

79.4

5.59

81.1

*Any quantity $<0.005 \mathrm{wt}$. \% was reported trace.

\section{ANALYSES}

The following analyses were made:

A. Acid feed

1. Total acid

2. Nitric acid

B. Acid product

1. Total acid

2. Nitric acid

3. Nitrous acid

C. Organic product
1. Total acid
2. Nitric acid
3. Nitrobenzene
4. Dinitrobenzene (qualitative test)

benzene $(2,6)$. The nitric and sulfuric acid content of the organic product was determined from the wash water by the foregoing methods.

Neither nitrous acid nor dinitrobenzene was detected in any of the runs.

\section{EXPERIMENTAL RESULTS}

The effects of agitation, phase composition, phase ratio, and temperature on the rate of nitration of benzene with mixed acid were investigated. Among the thirty-four runs the agitator speed was
Tables 1 and 2 present the operating conditions and analytical results, respectively, which were calculated for the thirty-four runs. Material balances for the over-all material flow, sulfur, and nitrogen were calculated for each run. The maximum deviation for the nitrogen balance was $4.1 \%$. The majority of all the material balance deviations was less than $2.0 \%$. Detailed sample calculations of the experimental data recorded in Tables 1 and 2 are available (1). 


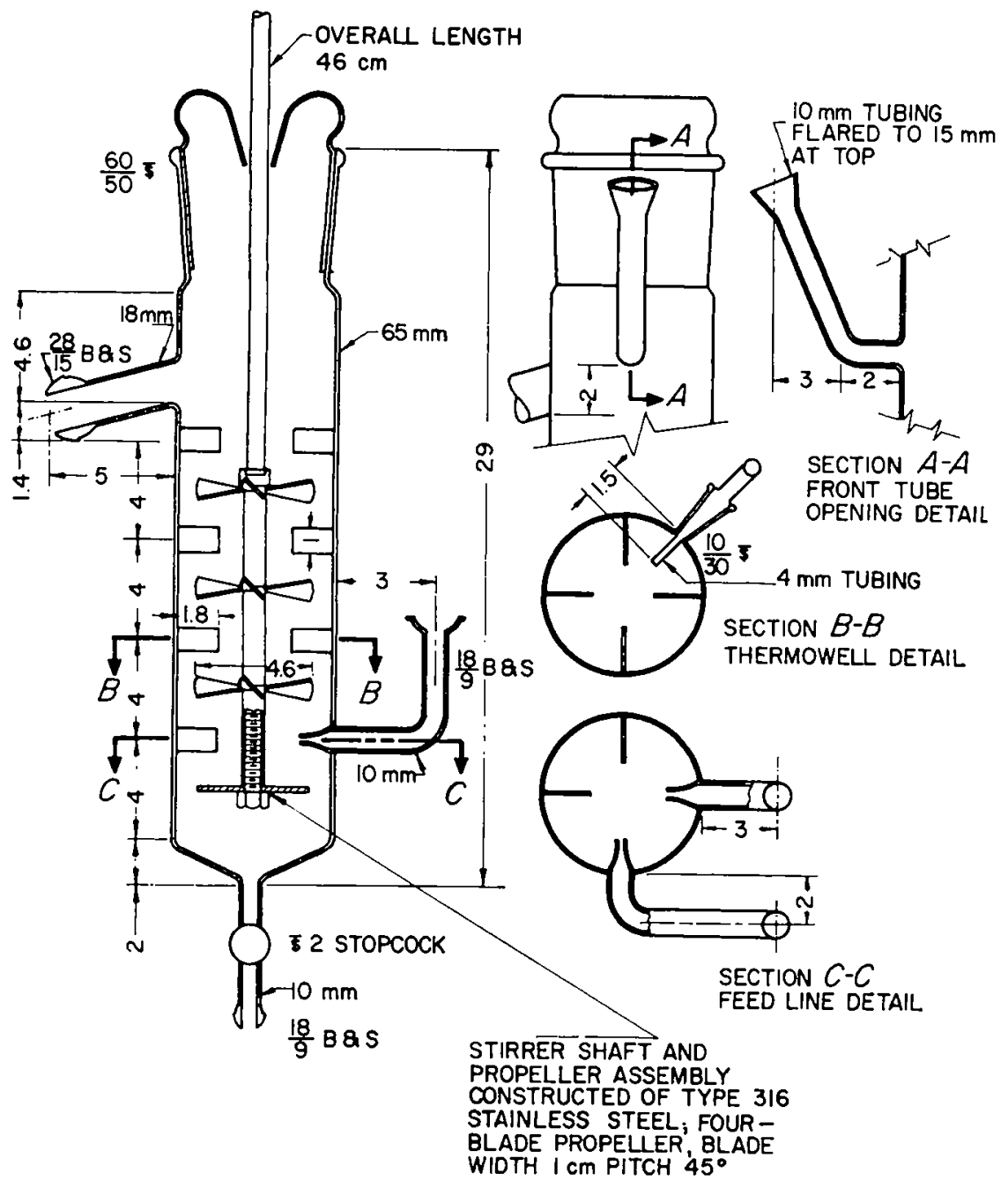

\section{DISCUSSION}

Test for Steady State Conditions

Run 1 served to justify the basis for steady state conditions used for the majority of the runs. After the densities of two successive organic samples agreed within the set limits for steady state operation, samples were taken of the organic and the acid products. The operation of the equipment was continued for an additional hour, whereupon a second set of samples was collected.

The uniformity of the emulsion in the reactor during steady state operation is indicated by the agreement for most of the runs of the volume percentage of acid phase measured in the reactor and that calculated from the product streams. Further evidence of uniformity was the agreement of the analyses of acid-phase samples from the reactor and from the acid-product stream.

Effect of Agitation

The over-all reaction between benzene and nitric acid in mixed acid is the sum of the homogeneous reactions in the acid and the organic phases. Previous studies of aromatic nitration $(11,12)$ indicated that the reaction is essentially irreversible; therefore, the over-all reaction may be written

$$
\begin{aligned}
& R=R_{A}+R_{O} \\
& R=k_{k_{A}} a_{A N} a_{A B} \frac{V_{A}}{100}+k_{O} a_{O N} a_{O B} \frac{V_{O}}{100}
\end{aligned}
$$

Fig. 2. Reactor.

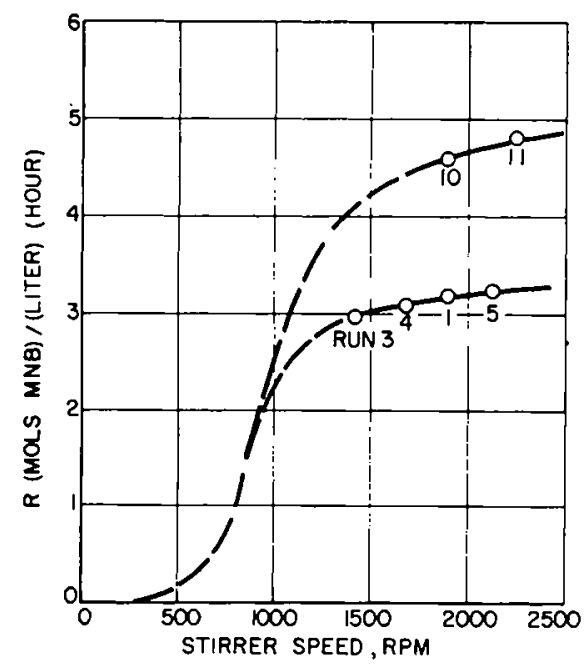

Fig. 3. Effect of agitation upon reaction rate.

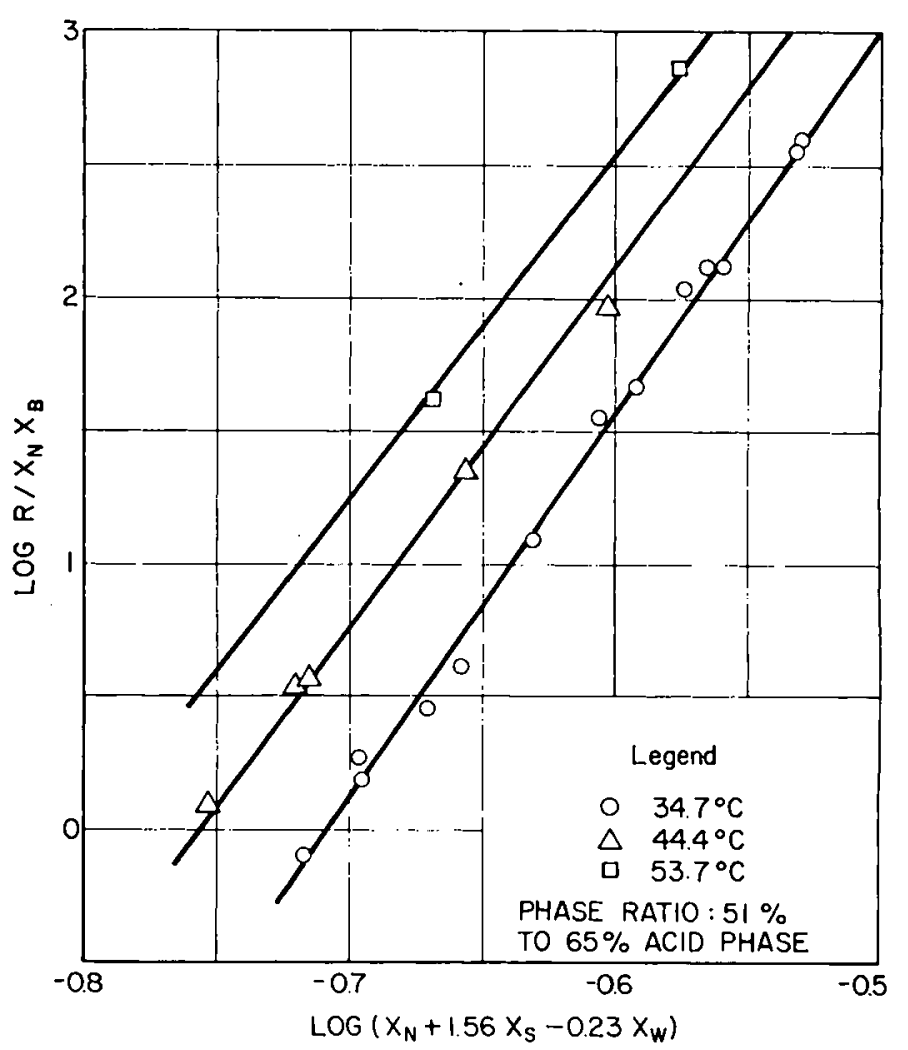

Fig. 4. Reaction-rate-composition correlation. 
Under steady state conditions benzene transfers into the acid phase at the same rate as it is consumed in that phase by chemical reaction. Similarly, the nitric acid transfers into the organic phase at the same rate as it is consumed in the organic phase. This situation can be expressed mathematically as

$$
\begin{aligned}
& R_{O}=K_{N}\left(a_{A N}-a_{O N}\right) \\
& R_{A}=K_{B}\left(a_{O B}-a_{A B}\right)
\end{aligned}
$$

Equation (3) represents the mass transfer of nitric acid, and Equation (4) represents the mass transfer of benzene.

Substituting Equations (3) and (4) into (2) gives

$$
\begin{aligned}
R= & {\left[k_{A} a_{A N}\left(a_{O B}-\frac{R_{A}}{K_{B}}\right)\right] \frac{V_{A}}{100} } \\
& +\left[k_{O} a_{O B}\left(a_{A N}-\frac{R_{O}}{K_{N}}\right)\right] \frac{V_{O}}{100}
\end{aligned}
$$

Since the terms in the brackets in Equation (5) represent $R_{A}$ and $R_{O}$ respectively, Equation (5) may be written

$$
\begin{aligned}
R=\frac{a_{A N} a_{O B}}{100}[ & \frac{V_{A}}{\left(\frac{1}{k_{A}}+\frac{a_{A N}}{K_{B}}\right)} \\
& \left.+\frac{V_{o}}{\left(\frac{1}{k_{O}}+\frac{a_{O B}}{K_{N}}\right)}\right]
\end{aligned}
$$

Increasing the degree of agitation by nereasing the stirrer speed will reduce the resistance terms to mass transfer in (6) by increasing the transfer coefficients $K_{B}$ and $K_{N}(5, \gamma)$. Thus if the stirrer speed is increased until the terms $a_{A N} / K_{B}$ and $a_{O B} / K_{N}$ become negligible with respect to $1 / k_{A}$ and $1 / k_{o}$ respectively, Equation (6) reduces to

$$
R=a_{A N} a_{O B} \frac{\left(k_{A} V_{A}+k_{o} V_{o}\right)}{100}
$$

which gives the over-all reaction rate expressed in terms of activities of nitric acid and benzene in the acid and organic phases respectively.

As $a=\gamma X$ Equation (7) may be written in terms of activity coefficients and mole fractions as

$R=X_{A N} X_{O B} \gamma_{A N} \gamma_{O B}\left(\frac{k_{A} V_{A}+k_{o} V_{O}}{100}\right)$

also as

$$
\begin{gathered}
\frac{V_{O}}{100}=1-\frac{V_{A}}{100} \\
R=\left(X_{A N} X_{O B}\right)\left(\gamma_{A N} \gamma_{O B} k_{A}\right) \\
\cdot\left[\left(1-\frac{k_{O}}{k_{A}}\right) \frac{V_{A}}{100}+\frac{k_{O}}{k_{A}}\right]
\end{gathered}
$$

The effect of agitation on the reaction rate is illustrated in Figure 3, where the reaction rates for runs $1,3,4,5,10$, and 11 are plotted against the stirrer speed. One curve is for the reactor in which runs 1 through 9 were made and the second curve is for the reactor (illustrated in Figure 2) in which the remainder of the runs were made. Each curve represents

\section{CORRELATION OF REACTION RATES}

Composition Correlation

Equation (9) suggests that if mass transfer between the phases is eliminated as a variable, the reaction rate can be correlated as a function of temperature, the nitric acid concentration in the acid phase, the benzene concentration in the organic phase, the volume percentage of

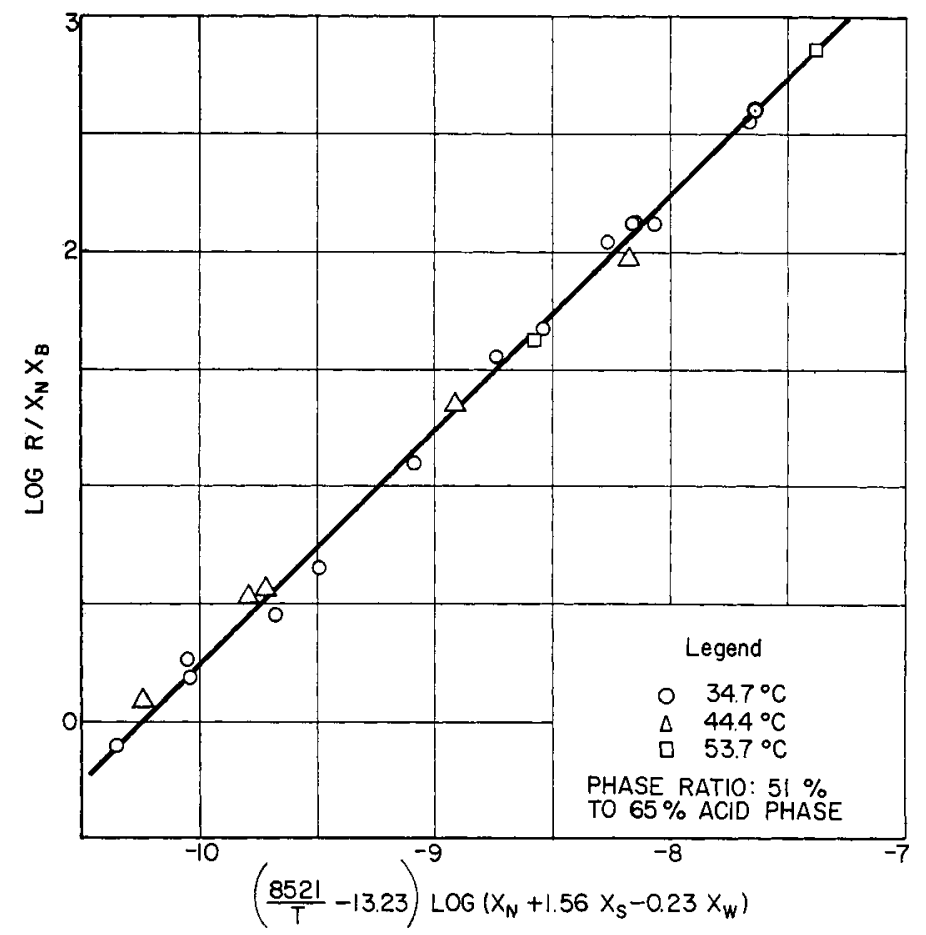

Fig. 5. Reaction-rate-composition-temperature correlation.

approximately constant phase compositions and phase ratio.

Figure 3 indicates that above 2,200 rev./min. in either reactor the resistance terms to mass transfer were made sufficiently small to eliminate the stirrer speed as a variable affecting the reaction rate. The great majority of the thirty-four runs were made at stirrer speeds above $2,200 \mathrm{rev} . / \mathrm{min}$. acid phase, and some function of the phase compositions. The latter variable is included because, in general, the term $\left(\gamma_{A N} \gamma_{O B}\right)$ is a function of composition.

In Figure 4 the values of $\log R / X_{N} X_{B}$ are plotted as a function of $\log \left(X_{N}+\right.$ $\left.1.56 X_{S}-0.23 X_{W}\right)$ for all the data with a volume percentage of acid phase between 50 and $65 \%$. All the data points at a common temperature fall close to a 


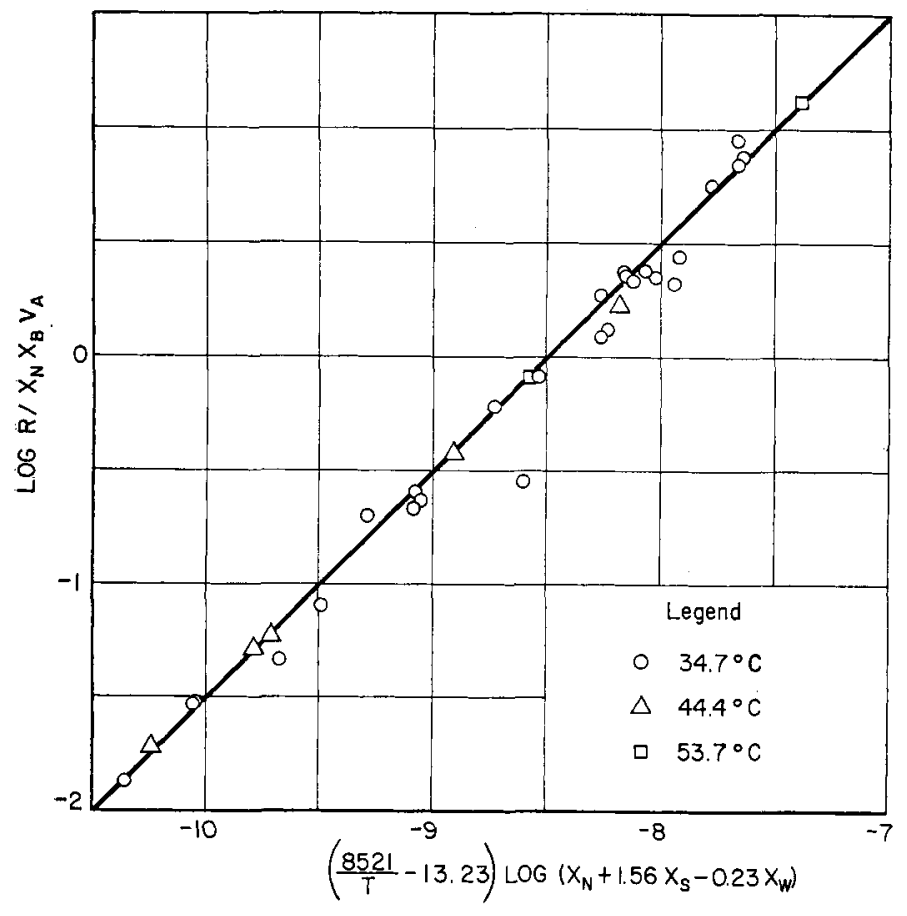

Fig. 7. Correlation including effect of phase ratio as a linear function.

straight line represented by the equation

$$
\begin{aligned}
\log \frac{R}{X_{N} X_{B}} & =P \log \left(X_{N}+1.56 X_{S}\right. \\
& \left.-0.23 X_{W}\right)+\log I
\end{aligned}
$$

or equal to zero. This general form of correlation was previously used to relate the rate of nitration of toluene with the phase compositions (B).

The empirical function $\left(X_{N}+1.56 X_{S}\right.$ $\left.-0.23 X_{W}\right)^{P}$ correlates the effect of the temperature as well as an effect of composition and can be considered as representing the product of the activity coefficients of benzene and nitric acid and the rate constant $k_{A}$, as indicated by Equation (9). The term

$$
\left[\left(1-\frac{k_{o}}{k_{A}}\right) V_{A}+\frac{k_{O}}{k_{A}}\right]
$$

of Equation (9) is insensitive to temperature over the $6 \%$ variation in absolute temperature covered in this investigation. Furthermore, the effect of phase ratio on the reaction rate indicates that $k_{O}$ is probably small as compared with $k_{A}$.

The correlation was presented in terms of the mole fraction of benzene in the organic phase on an acid-free basis for convenience only, as it makes little difference in the correlation whether the actual mole fraction or the acid-free mole fraction is used.

\section{Composition-temperature Correlation}

The three straight lines in Figure 4 may be extrapolated to a common intercept, and the slope $P$ of each line is found to be a function of temperature. The family of curves can be represented by the equation

where $P$ is the slope of the line, a function of temperature, and $\log I$ is the intercept when the straight line is extrapolated to a value of $\log \left(X_{N}+1.56 X_{S}-0.23 X_{W}\right)$

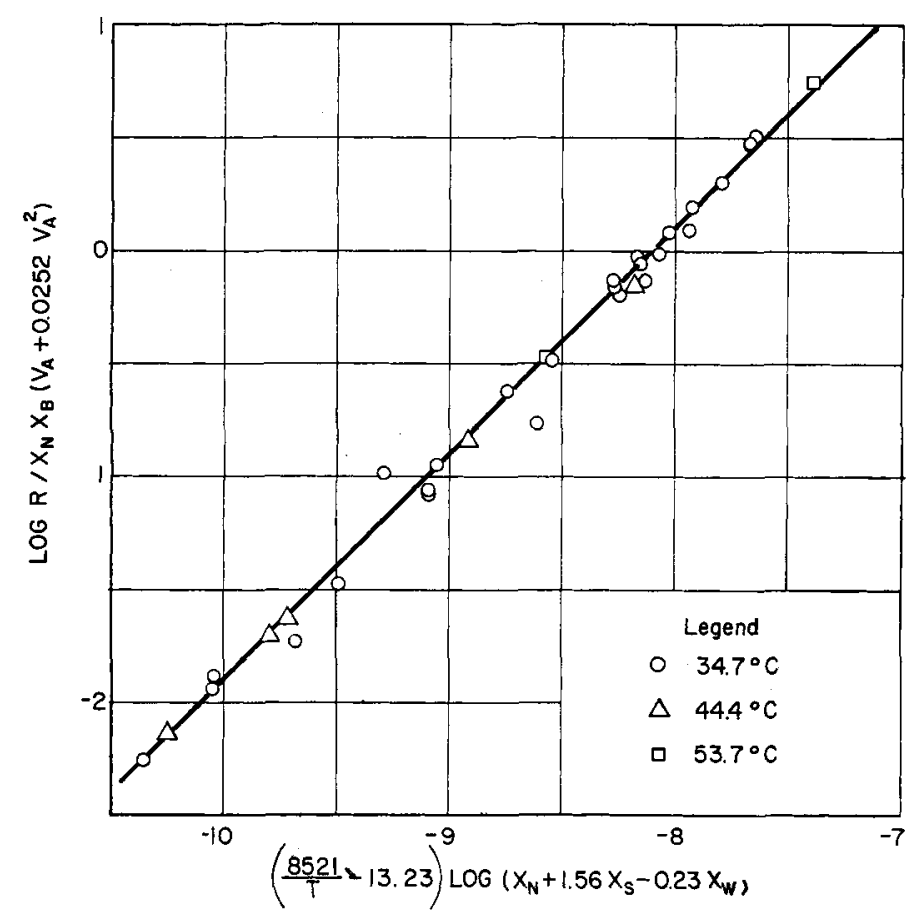

Fig. 8. Correlation including effect of phase ratio as a quadratic function.

$$
\begin{aligned}
& \log \frac{R}{X_{N} X_{B}}=\left(\frac{8521}{T}-13.23\right) \log \left(X_{N}\right. \\
& \left.+1.56 X_{S}-0.23 X_{W}\right)+10.24(12)
\end{aligned}
$$

Equation (12) can be rearranged to give

$R=\left(1.73 \times 10^{10}\right) X_{N} X_{B}$

.$e^{8521 / T-13.23 \ln \left(X_{N}+1.56 X_{S}-0.23 X_{W}\right)}$

The exponential function in Equation (13) is an empirical representation of the product $\left(\gamma_{A N} \gamma_{O B} k_{A}\right)$ in Equation (9).

The data in Figure 4 are represented as a single straight line in Figure 5 by Equation (12). As previously noted, Equations (12) and (13) are valid only for a volume percentage of acid phase in the range from 50 to $65 \%$ acid phase.

\section{Effect of Phase Ratio}

In an attempt to evaluate the extent of the nitration reaction in each phase, the volume percentage of acid phase was varied from 25 to 79 . It was impractical to hold the phase composition constant while the relative amounts of the phases were varied, and it became necessary to correct the data for these differences. It was assumed that the effect of composition and temperature at constant phase ratio was expressed by Equation (12) and that the effect of phase ratio could be accounted for as in Equation (9); the resulting expression was 
$R=x_{N} x_{B}\left[\left(x_{N}+1.56 x_{S}\right.\right.$

$$
\begin{aligned}
& \left.\left.-0.23 x_{W}\right)^{8521 / T-13.23}\right] \\
& \cdot\left[\left(1-\frac{k_{O}}{k_{A}}\right) V_{A}+\frac{k_{O}}{k_{A}}\right]
\end{aligned}
$$

The quantity $R / x_{N} x_{B}\left(x_{N}+1.56 x_{S}-\right.$ $\left.0.23 x_{W}\right)^{8621 / T-13.23}$ was plotted against the volume percentage of acid phase $V_{A}$ in Figure 6.

According to Equation (14), the data plotted in Figure 6 should fall on a straight line, the intercept being equal to $k_{O} / k_{A}$ and the slope being equal to $\left(1-k_{O} / k_{A}\right)$. This means that the slope can take on any value, positive or negative, and that the intercept must be greater than or equal to zero.

The straight line which most nearly satisfies the theoretical requirements is labeled as curve $A$ on Figure 6. This indicates that the reaction rate in the organic phase is negligibly small compared with the reaction rate in the acid phase. Figure 7 shows a final correlation based on curve $A$ of Figure 6 . The equation of the straight line representing the data in Figure 7 is

$R=\left(3.12 \times 10^{8}\right) x_{N} x_{B} V_{A}\left(x_{N}\right.$

$$
\left.+1.56 x_{S}-0.23 x_{W}\right)^{8521 / T-13.23}
$$

Although it was contrary to Equation (14), a parabola, curve $B$, was drawn through the data in Figure 6. Curve $B$ gives a better representation of the data below, and a satisfactory representation of the data above, $50 \%$ acid phase. Figure 8 shows a final correlation based on curve $B$ of Figure 6 . The equation for the correlation of Figure 8 is

$$
\begin{aligned}
R= & \left(1.233 \times 10^{8}\right) x_{N} x_{B}\left(V_{A}\right. \\
& \left.+0.0252 V_{A}^{2}\right)\left(x_{N}+1.56 x_{S}\right. \\
& \left.-0.23 x_{W}\right)^{8521 / T-13.23}
\end{aligned}
$$

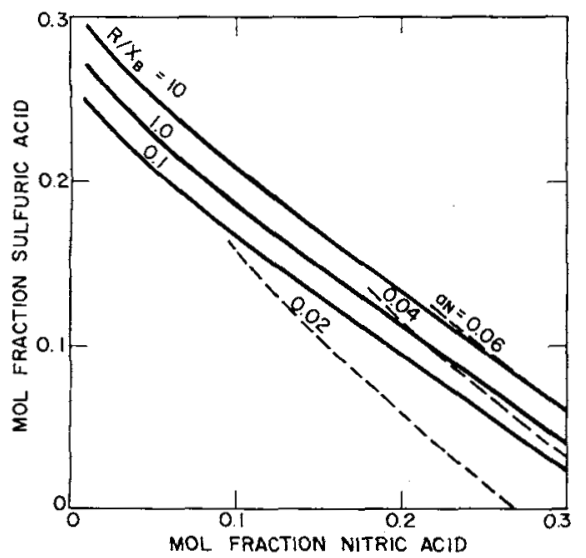

Fig. 9. Comparison of activity of nitric acid with rate of nitration of benzene.
Suen (16) reports that in the emulsion the acid phase is continuous above $33 \%$ acid phase and the organic phase is continuous below $33 \%$ acid phase. This inversion of phases might explain the fact that the data points below $50 \%$ acid phase in Figure 6 fail to substantiate function of acid-phase composition were drawn through the data. Equation (17) assumes that the vapor phase is an ideal gas and fixes the standard state fugacity as the fugacity of the pure component at the temperature of the mixture.

The rate of nitration of benzene is com-

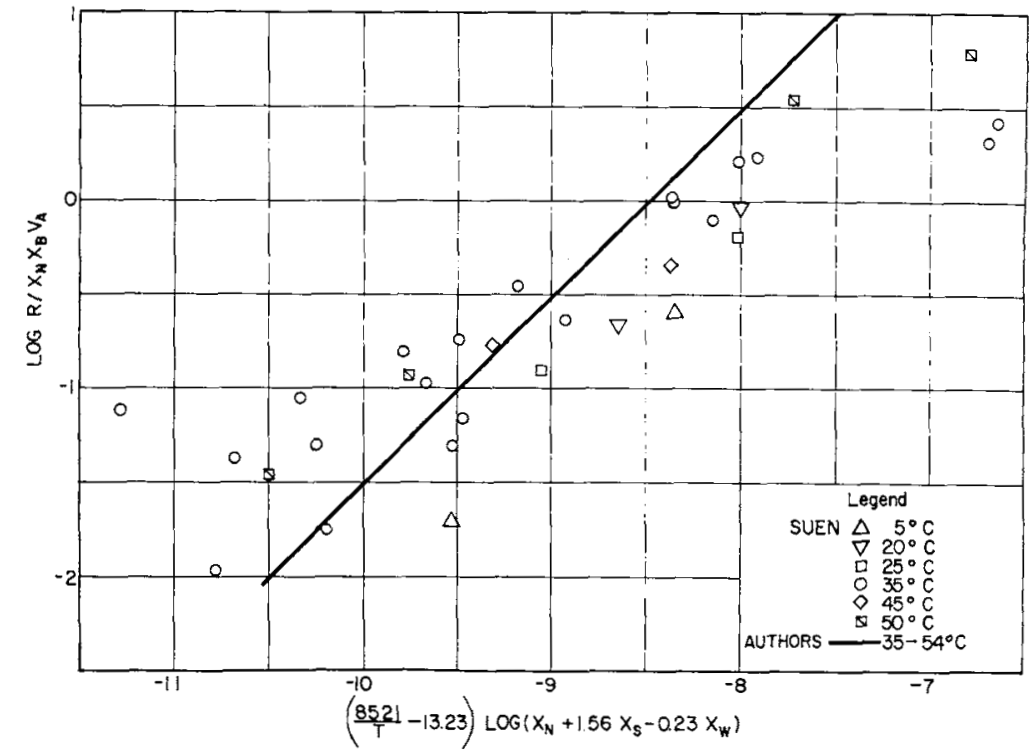

Fig. 10. Comparison of correlation with batch data of Suen.

Equation (14). It is possible that the agitation supplied failed to produce a sufficiently fine emulsion near the inversion point to eliminate completely the mass transfer effects.

However, three runs made at a volume percentage of acid phase from 30 to $33 \%$ and at stirrer speeds from 2,260 to 2,810 $\mathrm{rev} . / \mathrm{min}$. gave consistent reaction rates as illustrated in Figure 6 . This agreement of rates indicates that the stirrer speed had no significant effect on the reaction rates in this range of volume percentage of acid phase.

Equation (16) is empirical; however, the function

$\left(X_{N}+1.56 X_{S}-0.23 X_{W}\right)^{8521 / T-13.23}$

can be considered to represent the term $\left(\gamma_{A N} \gamma_{O B} k_{A}\right)$ in the theoretical Equation (9). Activity coefficients must be evaluated by independent equilibrium measurements to verify this viewpoint, but only a limited amount of the necessary data is available.

Lewis and Suen (11) reported some vapor-liquid equilibrium measurements for nitric acid-sulfuric acid-water mixtures at $35^{\circ} \mathrm{C}$. over a limited range of liquid-phase compositions. Activities of nitric acid in these mixtures were calculated according to the equation

$$
A=\frac{P y}{p}
$$

and the dashed curves in Figure 9 illustrating the activity of nitric acid as a pared with the activity of nitric acid in Figure 9 where curves of constant $R / X_{B}$ as calculated from Equation (16) for $35^{\circ} \mathrm{C}$. and $60 \%$ acid phase are drawn as the solid lines. The rate of nitration appears to be a function of the activity of nitric acid although the relationship is not first order, as assumed in the theoretical equations. No further conclusions can be drawn from the comparison in Figure 9 because of the limitations of the equilibrium data.

\section{Comparison of Correlation with Suen's Data}

Reaction-rate data for the nitration of benzene with mixed acid was determined by Suen (16) in a series of batch experiments. A comparison of Suen's data with those of the authors is illustrated in Figure 10, where rates calculated from the conversion-time data reported by Suen are plotted with Equation (15).

Although all the data reported by Suen were not used in calculating the results plotted, Figure 10 is considered to be an objective representation of his results. A tabulation of these calculated rates is available (1).

Lewis and Suen (11) report, in addition to the batch data, the results from a study of the effect of phase ratio, or volume percentage of acid phase, in a continuous apparatus. These results indicate that there is a significant reaction rate in the organic phase relative to that in the acid phase. Values estimated from a published figure (11) are shown as the 


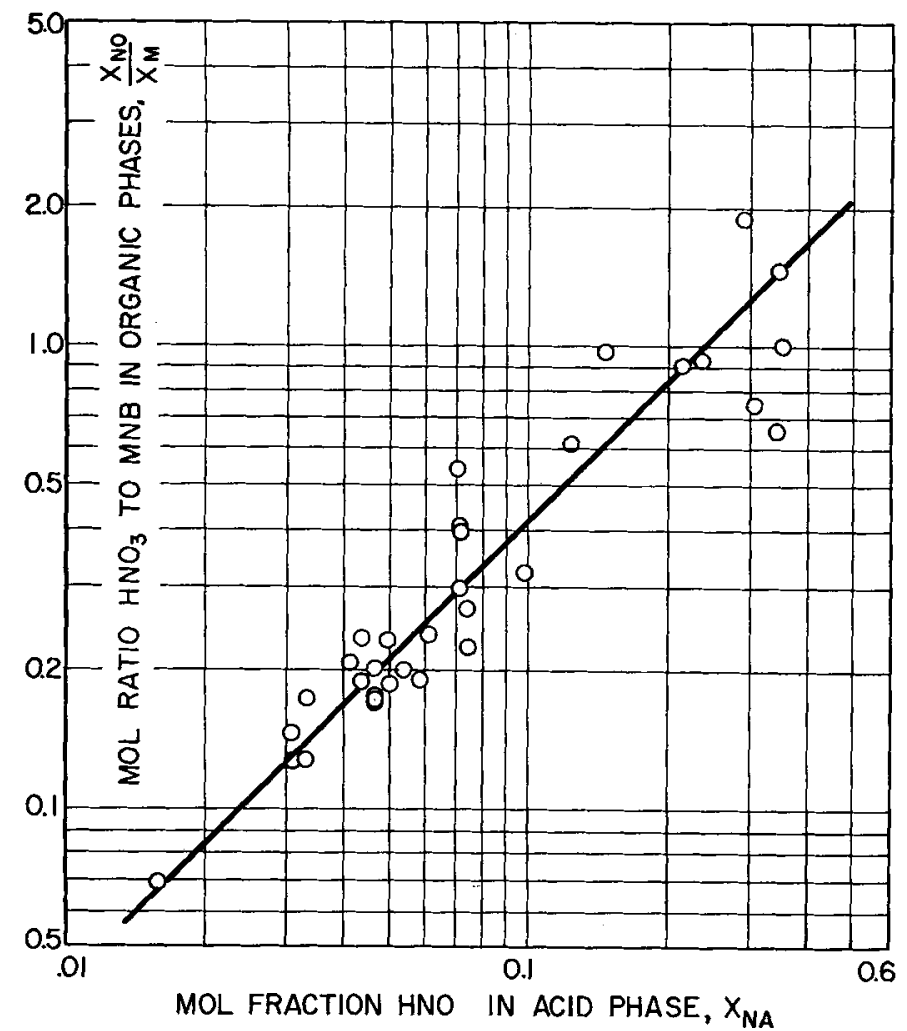

Fig. 11. Distribution of nitric acid between acid and organic phases. Equation of line: $X_{N O} / X_{M N B}=8.4 X_{N A}$. dotted line (curve $C$ ) of Figure 6. No explanation of this discrepancy can' be offered since neither the original data nor the equipment details were published.

\section{Comparison with Toluene Nitration Data}

The data of this investigation permit a quantitative comparison with the rate of nitration of toluene $(12)$. At $35^{\circ} \mathrm{C}$. the rate of nitration of toluene under comparable conditions of nitration ranges from 1.76 to 9.7 times faster than the rate of nitration of benzene in the range of acid-phase compositions studied.

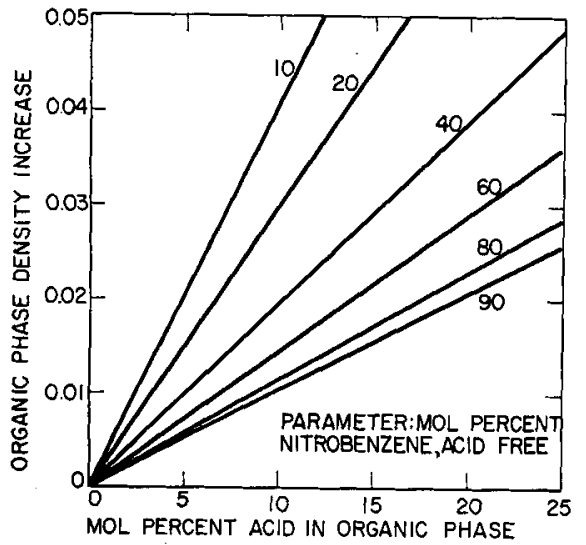

Fig. 12. Effect of dissolved acid on density of organic phase.
$B=$ constant

$e=2.718$

$I=$ constant

$K_{B}=$ mass transfer coefficient for benzene transfer, g.-moles/(hr.) (liter)

$K_{N}=$ mass transfer coefficient for nitric acid transfer, g.-moles/(hr.) (liter)

$k_{A}=$ reaction velocity constant for acidphase reaction

$k_{o}=$ reaction velocity constant for organic-phase reaction

$P=$ constant

$R=$ reaction rate, g.-moles/(hr.)(liter)

$T=$ temperature, ${ }^{\circ} \mathrm{K}$.

$V_{A}=$ volume percentage of acid phase

$V_{0}=$ volume percentage of organic phase

$x_{A N}, x_{N}=$ mole fraction of nitric acid in acid phase

$x_{B}=$ mole fraction of benzene in organic phase, acid-free basis

$x_{M}=$ mole fraction of nitrobenzene in organic phase, acid-free basis

$x_{O B}=$ mole fraction of benzene in organic phase

$x_{O N}=$ mole fraction of nitric acid in organic phase

$x_{S}=$ mole fraction of sulfuric acid in acid phase

$x_{W}=$ mole fraction of water in acid phase

$\gamma_{A N}=$ activity coefficient of nitric acid in acid phase

$\gamma_{O B}=$ activity coefficient of benzene in organic phase

\section{IITERATURE CITED}

1. Biggs, R. D., Ph.D. thesis, Univ. Mich., Ann Arbor (1954).

The rate of nitration of benzene with mixed nitric acid and sulfuric acid has been measured over ranges of phase composition, temperature, and phase ratio that are of interest in commercial nitration. Although it is not clear whether or not the effects of mass transfer have been completely eliminated for all values of the phase ratio investigated, the data do in all probability represent limiting values of the reaction rate obtainable by ordinary means of agitation. For this reason Equation (16) is recommended for use in design calculations, but it should be used with caution in view of the difficulty of obtaining the same high degree of agitation in large-scale reactors.

The distribution of nitric acid between the acid and organic phases is shown in Figure 11, and the effect of dissolved acid on the density of the organic phase is shown in Figure 12. The accuracy of the data upon which Figures 11 and 12 are based is not high, but they are useful for the purpose of estimating these effects.

\section{NOTATION}

$A=$ constant

$a_{A B}=$ activity of benzene in acid phase

$a_{A N}=$ activity of nitric acid in acid phase

$a_{O B}=$ activity of benzene in organic phase

$a_{O N}=$ activity of nitric acid in organic phase
2. Bost, R. W., and F. Nicholson, Ind. Eng. Chem., Anal. Ed., 7, 190 (1935).

3. Crooks, R. C., and R. R. White, Chem. Eng. Progr., 46, 249 (1950).

4. Hann, J. W., and K. A. Kobe, Ind. Eng. Chem., 43, 2355 (1951).

5. Hixson, A. W., and M. I. Smith, Ind. Eng. Chem., 41, 973 (1949).

6. Jurecek, M., Chem. Listy, 43, 204 (1949).

7. Karr, A. E., and E. G. Scheibel, Chem. Eng. Progr. Symposium Series No. 10, 50,73 (1954).

8. Kobe, K. A., and J. J. Mills, Ind. Eng. Chem., 45, 287 (1953)

9. Kobe, K. A., and P. W. Pritchett, Ind. Eng. Chem., 44, 1398 (1952).

10. Kolthoff, I. M., E. B. Sandell, and B. Moskovitz, J. Am. Chem. Soc., 55, 1454 (1933).

11. Lewis, W. K., and T. J. Suen, Ind. Eng. Chem., 32, 1095 (1940).

12. McKinley, C., and R. R. White, Trans. Am. Inst. Chem. Engrs., 40, 143 (1944).

13. Mitscherljch, E., Ann. Physik u. Chem., 31, 625 (1834).

14. Othmer, D. F., J. J. Jacobs, Jr., and J. F. Levy, Ind. Eng. Chem., 34, 286 (1942).

15. Scott, W. W., "Scott's Standard Methods of Chemical Analysis," Vol. 2, 5 ed., 2212-13, 2234, D. Van Nostrand Company, Inc., New York (1939).

16. Suen, T. J., Sc.D. thesis, Mass. Inst. Tech., Cambridge (1937).

Presented at A.I.Ch.E. New York meeting 